\title{
ESTUDIO DE LA PRODUCCIÓN CIENTÍFICA SOBRE SOCIAL MEDIA. EL CASO DE LAS REVISTAS ESPAÑOLAS DE COMUNICACIÓN EN JCR Y SJR
}

\section{THE STUDY OF ACADEMIC OUTPUT ON SOCIAL MEDIA RESEARCH TOPIC IN JCR AND SRJ JOURNALS SPECIALIZED IN COMMUNICATION IN SPAIN}

Emilia Smolak Lozano'. Universidad de Málaga. España. esmolaklozano@uma.es.

(8) $\mathbb{R}^{\mathrm{C}}$ Ana Almansa-Martínez². Universidad de Málaga. España anaalmansa@uma.es

\section{Cómo citar el artículo:}

Smolak Lozano, E. y Almansa-Martínez, A. (2021). Estudio de la producción científica sobre social media. El caso de las revistas españolas de comunicación en JCR y SJR. Revista de Ciencias de la Comunicación e Información, 26, 15-38. http://doi.org/10.35742/rcci.2021.26.e124

\section{RESUMEN}

La presencia de los Social Media en la difusión de la investigación científica española es el objeto de este estudio. Concretamente, se centra en las revistas españolas indexadas en JCR y SJR: Comunicar, Comunicación y Sociedad, El Profesional de Información, SCIRE, Signa, Revista Latina de Comunicación, Ibersid, Historia y Comunicación Social y Estudios sobre el Mensaje Periodístico. Se ha realizado un análisis de contenido de 2.427 artículos para el periodo 2012-2016, ilustrando la madurez en el desarrollo y la implementación de las redes sociales en la comunicación humana.

PALABRAS CLAVE: redes sociales; comunicación; revistas científicas, bibliometría, investigación.

\section{ABSTRACT}

\footnotetext{
${ }^{1}$ Emilia Smolak Lozano. Universidad de Málaga: esmolaklozano@uma.es. Doctora en ciencias de la Comunicación, docente e investigadora en publicidad y relaciones públicas, con un enfoque a la aplicación de las nuevas tecnologías.

2 Ana Almansa Martínez. Universidad de Málaga: anaalmansa@uma.es. Profesora Titular de Universidad, doctora en ciencias de la Comunicación, docente e investigadora en dirección de comunicación, relaciones públicas y comunicación política.
}

Recibido: 09/02/2021 - Aceptado: 15/04/2021 - Publicado: 05/05/2021 
The object of the study of this article is the presence of Social Media in the dissemination of Spanish scientific research. In particular, it focuses on the Spanish journals included in JCR and SJR rankings: Comunicar, Comunicación y Sociedad, The Professional of Information, SCIRE, Signa, Latina Journal of Social Communication, Ibersid, History and Social Communication and Studies on the Journalistic Message. There has been carried out the content analysis of 2,427 articles during the period of 2012 and 2016, illustrating the maturity in the development and implementation of social networks in human communication.

KEYWORDS: social networks; communication; scientific journals; investigation, bibliometric studies.

\section{INTRODUCCIÓN}

Las revistas científicas son el instrumento principal de difusión de las investigaciones para que sean conocidas por la comunidad científica. En consecuencia, analizar los contenidos de las revistas permite conocer el tipo de investigaciones que se están realizando en un campo científico o sobre determinadas temáticas.

En este sentido, las revistas son el principal instrumento de difusión de las investigaciones tanto dentro de la academia como en la sociedad. Es posible conocer la evolución de un campo científico a través del análisis de sus textos en las revistas de referencia, ya sea mediante aspectos temáticos, de fuentes, de autoría, de género o de procedencia de sus autores, entre otras cuestiones (Canaza-Choque, 2018).

En este artículo se analiza, en concreto, la línea de investigación de los Social Media, en las principales revistas españolas del campo de la Comunicación, para conocer la tipología, temporalidad y modalidad de las investigaciones. Todo ello enmarcado en las características de la Comunicación, con una alta trans-disciplinaridad (Repiso, Torres-Salinas y Delgado, 2016), lo que le confiere la posibilidad de mostrar el estudio de los Social Media desde diferentes ámbitos disciplinarios y sobre diversos objetos de estudio, como se puede ver en caso de la obra sobre uso, tipología y consumo de las redes sociales (Caldevilla Domínguez, 2010).

Las investigaciones demuestran que el "predominio académico-universitario resulta aplastante frente a aquellos autores vinculados a sectores profesionales 0 instituciones diferentes a la Universidad" (Castillo-Esparcia, Rubio-Moraga y AlmansaMartínez, 2012). Es decir, van a ser principalmente académicos quienes realizan publicaciones en revistas. Además, entre estos investigadores-académicos suele ser habitual una colaboración interuniversitaria, de manera que "en aquellos artículos en los que figuran más de dos autores la procedencia de estos suelen corresponder a Universidades o Instituciones distintas" (Castillo-Esparcia, Rubio-Moraga y AlmansaMartínez, 2012).

La investigación sobre las revistas en España ha despertado gran interés en la última década, en la que se han venido realizando publicaciones sobre este ámbito (Martínez-Nicolás y Saperas-Lapiedra, 2011; Giménez-Toledo 2013; Montero-Díaz, Cobo, Gutiérrez-Salcedo, Segado-Boj y Herrera-Viedma, 2018; Castillo-Esparcia y 
Carretón Ballester, 2010; Torres-Salinas, Delgado-López-Cózar, García-MorenoTorres y Herrera, 2011; Repiso-Caballero y Torres-Salinas, 2014; De Filippo, 2013; De Pablos-Coello, Túñez-López y Mateos-Martín, 2015), especialmente en el caso de la Comunicación.Y desde la perspectiva de que se incluyen textos teóricos, pero también aplicados, a las diferentes tipologías organizativas, como pueden ser empresas, instituciones públicas, asociaciones sociales, partidos políticos, entre otros (Almansa-Martínez y Castillo-Esparcia, 2014). Los Social Media han vivido su desarrollo en los diferentes ámbitos profesionales, siendo en la actualidad una parte importante en la estrategia comunicativa de los diversos tipos de organizaciones (Estudio Anual de Redes Sociales, 2019).

Esa interdisciplinariedad e interrelaciones entre diferentes organizaciones influye en el cómo se están aplicando los Social Media, dónde y desde cuándo. Así, podemos establecer una radiografía del grado de inserción de los Social Media en las estrategias de comunicación de las organizaciones y personas (Barón Pulido et al., 2021); y si esa implicación se ha producido con rapidez o no, mediante el estudio de cuándo está disponible la aplicación digital y el tiempo de uso de las organizaciones. Los Social Media se han convertido, de esta manera, en un claro objeto de estudio de interés tanto profesional y como científico.

Disponer de información sobre un determinado ámbito de estudio coadyuva a que en el campo científico sea posible conocer las líneas de investigación emergentes entre sus investigadores (Torres-Salinas, Jiménez-Contreras y Robinson-García, 2014; Park y Leydesdorff, 2009), puesto que la Comunicación se muestra como una disciplina interrelacionada con diferentes contextos científicos, como son la economía, la sociología o las ciencias políticas (Leydesdorff y Probst, 2009).

Los Social Media han tenido en la última década una gran implementación social, en general, y profesional, en particular, convirtiendo asimismo al ciudadano como protagonista de comunicación como demuestran estudios bibliográficos sobre este tema (Landeta Bejarano et al., 2020). Por lo tanto, el estudio de los Social Media parece que debe ser una de las principales líneas de investigación en las revistas científicas, tenida en cuenta, además, la especial significación de la comunicación bidireccional en las estrategias de comunicación.

En este sentido, los primeros estudios (Boyd y Ellison, 2008) enfatizan los elementos teóricos, a la par que comienzan a incidir en las modificaciones del proceso de comunicación con el empoderamiento de todos los actores que participan en la acción comunicativa (Diga y Kelleher, 2009; Eyrich, Padman y Sweetser, 2008). Los estudios sobre el uso de los Social Media en Comunicación han proliferado en España en los últimos años, en las diferentes disciplinas y ámbitos (Rúas-Araújo y Casero-Ripollés, 2018; Ramos Soler, Del-Pino-Romero y Castello-Martínez, 2014; Castillo-Esparcia, Almansa-Martínez, Smolak-Lozano, 2015; Ure, 2016; Almansa-Martínez, Fonseca y Castillo-Almansa, 2013; Sánchez- Hervás, 2013; Rosero Molina et al., 2020).

\section{OBJETIVOS}

La principal pregunta de investigación que guía este estudio se centra en lo siguiente: ¿qué espacio ocupa la investigación de y en Social Media en artículos publicados en 
este lustro en las revistas indexadas en JCR y SRJ en España en el campo de las Ciencias de la Comunicación?

El objetivo de esta investigación es determinar, precisamente, el alcance de los Social Media en la difusión de la investigación científica española. Concretamente, la investigación se centra en las consideradas mejores revistas españolas de Comunicación, al entenderse que máxima calidad e impacto constituyen las pautas de desarrollo del campo de la Comunicación en España.

Partiendo de la premisa que los Social Media despiertan de forma creciente el interés científico y profesional en el campo de la Comunicación, se han determinado dos preguntas de investigación:

P1: ¿Los Social Media constituyen la mayor parte de la temática en la investigación

publicada en las revistas de impacto en Comunicación?

P2: ¿El alcance del interés por la investigación en los Social Media crece de forma exponencial a medida que se desarrollan y establecen las redes sociales como principales herramientas de Comunicación?

A partir de estas preguntas de investigación hemos planteado dos objetivos específicos para nuestro estudio:

OS1: Determinar alcance y grado de interés académico en Social Media en las publicaciones de alto impacto

OS2: Determinar la dirección y grado de crecimiento de este interés en función de la creciente popularidad de estas herramientas de comunicación en varios sectores.

\section{METODOLOGÍA}

Con el objetivo de cuantificar la producción científica en la temática de Social Media en la investigación en Comunicación publicada en las revistas de alto impacto, se han seleccionado las revistas Revistas españolas indexadas en JCR y SJR: Comunicar, Comunicación y Sociedad, Profesional de Información, SCIRE, Signa, Revista Latina de Comunicación Social, Ibersid, Historia y Comunicación Social y Estudios sobre el Mensaje Periodístico. El periodo de análisis han sido 5 años (2012-2016, ambos incluidos), siendo la época de desarrollo de las redes sociales- su desarrollo e implementación en la sociedad a su vez- entran en la fase de la madurez y consolidación en España y a nivel mundial.

La técnica de investigación empleada es una revisión y sistematización bibliográfica de carácter cuantitativo y cualitativo (Codina, 2018; Hernández Salazar, 2019), basada en el análisis de contenido y técnicas semánticas, mediante una plantilla de ítems creada para tal fin. La plantilla de análisis contiene los datos de identificación de la revista, tales como nombre de la revista, número, volumen, año de publicación. Estas variables nos facilitan de modo ilustrativo los periodos temporales cuando crezca el interés por el tema, así como los volúmenes más dedicados a los fenómenos 2.0 y las revistas que más publican sobre este campo. Elementos de la plantilla de la investigación eran los siguientes: 
Tabla 1. Plantilla de análisis

\begin{tabular}{|l|l|}
\hline Nombre de la revista & Título del artículo \\
\hline Número de la revista & Palabras clave \\
\hline Volumen & Año de publicación \\
\hline Año de publicación & Nombre y apellidos de autores \\
\hline Universidad & País de publicación \\
\hline País & Metodología \\
\hline
\end{tabular}

Fuente: elaboración propia

Con el instrumento utilizado en la investigación se ha analizado a través del buscador de Dialnet a cada página web de las revistas seleccionadas para comprobar la presencia de los textos que incluyen las palabras clave Social Media, medios sociales, medios digitales, medios sociales digitales, redes sociales y redes sociales digitales.

Una vez comprobada la existencia de los textos con al menos una de las palabras clave, hemos descargado el texto y hemos analizado el título del artículo, resumen (abstract), palabras clave (keywords) y texto completo, empleando la plantilla.

Tabla 2. Palabras clave utilizadas en búsqueda bibliométrica

\begin{tabular}{|l|l|}
$\begin{array}{l}\text { Palabras clave de búsqueda (Keywords) } \\
\text { principales }\end{array}$ & $\begin{array}{l}\text { Social Media } \\
\text { medios sociales }\end{array}$ \\
\hline $\begin{array}{l}\text { Palabras clave de búsqueda (Keywords) } \\
\text { secundarios }\end{array}$ & \begin{tabular}{l} 
medios digitales \\
medios sociales digitales \\
redes sociales \\
\hline
\end{tabular} \\
\hline
\end{tabular}

Fuente: elaboración propia

Dada la pluralidad y complejidad que existe en cuanto al uso de este término universalmente aplicado en su origen anglosajón (Social Media), se ha decidido contemplar todas estas denominaciones, además de los nombres específicos de redes sociales. También, ha sido de suma importancia mirar tanto el resumen como el texto para asegurar la validez y coherencia del estudio. Adicionalmente, el uso de técnicas bibliométricas nos permitió contrastar el volumen y su fluctuación de publicaciones sobre Social Media con la producción científica total en Comunicación y en las revistas indexadas. De este modo, desde Noviembre de 2017 hasta Diciembre de 2018 se ha llevado a cabo el análisis de las publicaciones desde 2012 hasta 2016 en las revistas indexadas, a través de sus versiones digitales. En total, la investigación abarcó 2427 artículos en las 9 revistas JCR y SJR anteriormente mencionadas ${ }^{3}$.

\footnotetext{
${ }^{3}$ Para aclarar el uso de los nombres de las revistas, se debe mencionar que por las razones de espacio en los gráficos y en las tablas se han acortado los nombres de las revistas de la siguiente manera: El Profesional de Información a EPI, Estudios sobre el Mensaje Periodístico aparece como ESMP y Revista Latina de Comunicación Social como Revista Latina. En otros casos se ha utilizado Com. en lugar de Comunicación siempre por razón de espacio y para fomentar la visibilidad de los datos y facilitar su lectura.
} 


\section{DISCUSIÓN}

Los resultados han demostrado, en primer lugar, el gran volumen de las publicaciones de investigación en Comunicación en los últimos 5 años que abarcan las revistas: 117 números acumulados en 38 volúmenes, con un total de 2427 artículos, tal como refleja la Tabla 3.

Tabla 3. Producción científica en las revistas indexadas 2012-2016

\begin{tabular}{|c|c|c|c|c|c|c|c|c|c|c|}
\hline \multirow{2}{*}{$\begin{array}{l}\text { Año } \\
\text { Nombre de la } \\
\text { revista }\end{array}$} & \multicolumn{10}{|c|}{ 2012-2016 } \\
\hline & 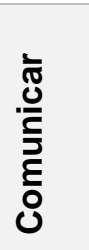 & \begin{tabular}{rl} 
& \multicolumn{2}{c}{} \\
$>$ & $\frac{\pi}{0}$ \\
$\varepsilon \dot{0}$ & $\frac{\pi}{0}$ \\
0 & 0 \\
0
\end{tabular} & $\overline{\mathbf{Q}}$ & 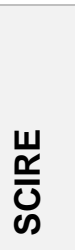 & $\frac{\pi}{5}$ & 吾 & 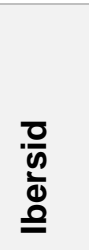 & 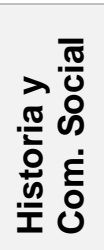 & $\sum_{\substack{n \\
w}}^{0}$ & $\begin{array}{l}\frac{\mathscr{d}}{\frac{\pi}{\pi}} \\
\frac{0}{\vdash}\end{array}$ \\
\hline $\begin{array}{l}\text { Volumen - } \\
\text { cantidad }\end{array}$ & 6 & 5 & 5 & 5 & na & $\mathrm{Na}$ & 7 & 5 & 5 & 38 \\
\hline $\begin{array}{l}\text { Número - } \\
\text { cantidad }\end{array}$ & 12 & 18 & 30 & 10 & 5 & 5 & 7 & 13 & 17 & 117 \\
\hline $\begin{array}{l}\text { Núm. Artículos } \\
\text { RRSS }\end{array}$ & 64 & 34 & 63 & 4 & 1 & 40 & 5 & 38 & 67 & 316 \\
\hline $\begin{array}{l}\text { Núm. Artículos } \\
\text { por Núm. Total }\end{array}$ & 200 & 192 & 381 & 86 & 173 & 213 & 100 & 507 & 575 & 2427 \\
\hline
\end{tabular}

Fuente: elaboración propia

Se puede apreciar en la figura 1 que los años 2012 y 2015 fueron los años con un menor número de publicaciones, aunque rodaron las 400, ligeramente por debajo de la media anual de producción científica para todas las revistas analizadas. La curva nos demuestra que la producción científica en revistas españolas varía mucho de un año a otro, sin una tendencia clara y definida. Destaca el pico de publicaciones que excede un 30\% la media anual, con 639 publicaciones en 2013 y 555 en 2014. Sin embargo, después se observa una caída en el año 2015 y un ligero crecimiento en el año 2016.

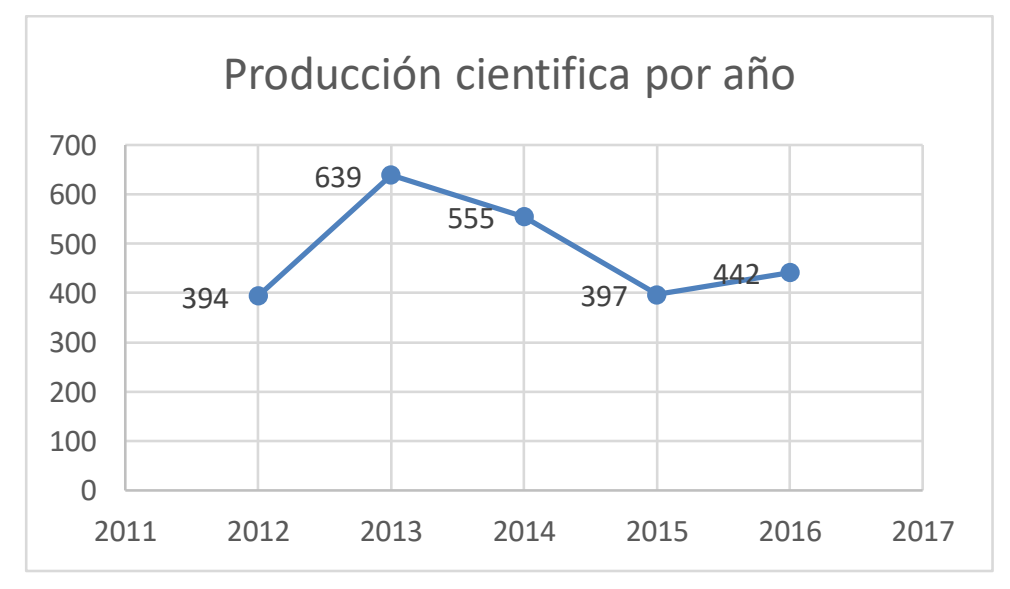

Gráfico 1. Producción científica por año

Fuente: elaboración propia 
Las revistas que más publican son la Historia y Comunicación Social y Estudio sobre el Mensaje Periodístico, al contar con el mayor número de volúmenes especiales. En tercer lugar, se ubica El Profesional de Información, con 381 publicaciones en el periodo analizado, lo que demuestra su impacto científico sobre la investigación en Comunicación.

La media en el período analizado de 5 años por revista es de 270 artículos, que es un número elevado de producción científica de alta calidad. La media de producción anual, por revista y año, que abarca este periodo de 5 años se detalla en la Tabla 3.

Se observa que Estudios sobre el Mensaje Periodístico cuenta con la mayor media de las revistas analizadas (115 artículos de media), seguida por Historia y Comunicación Social, con 101 y casi duplicando la producción anual en el mismo periodo de El Profesional de Información, con 76.2. Sólo Comunicar mantiene estable el número de publicaciones cada año, desde 2012 hasta 2016 (40 artículos cada año). SCIRE cuenta con una media del $50 \%$ por debajo de la de media anual para todas las revistas (20 artículos publicados frente a los más de 40 de media para todas las revistas en los años 2012, 2015 y 2016).

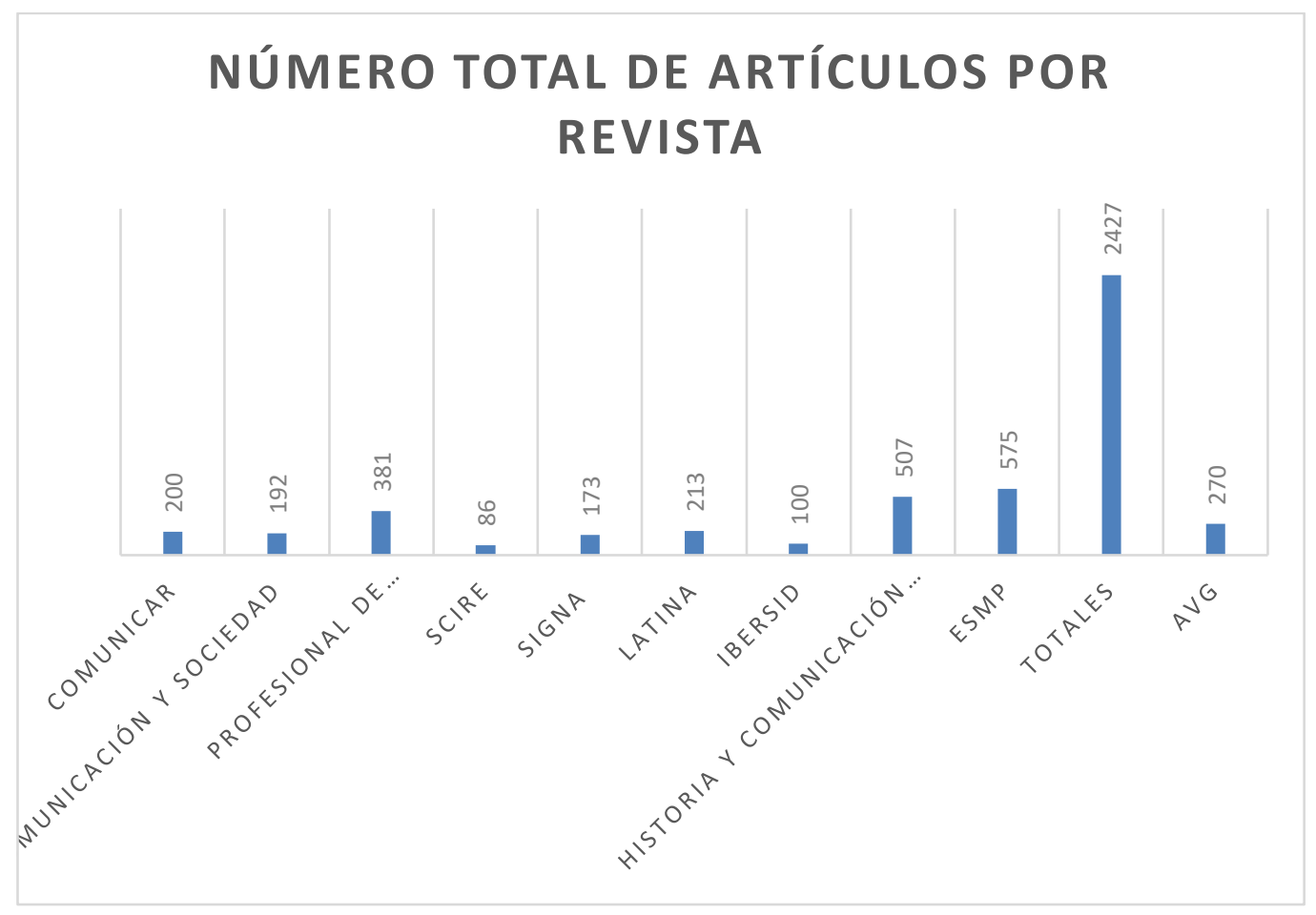

Gráfico 2. Producción científica 2012-2016 por revista

Fuente: elaboración propia

En general, en los últimos 5 años, la producción científica anual total ronda la media de 485 artículos. 
Tabla 4. Media por revista dentro del período de 5 años

\begin{tabular}{|l|r|}
\hline Numero artículos totales & AVG totales por año en el periodo y por revista \\
\hline Comunicar & 40 \\
\hline Comunicación y Sociedad & 38.4 \\
\hline El Profesional de Información & 76.2 \\
\hline SCIRE & 17.2 \\
\hline Signa & 34.6 \\
\hline Latina & 42.6 \\
\hline lbersid & 20 \\
\hline Historia y Comunicación Social & 101.4 \\
\hline ESMP & 115 \\
\hline
\end{tabular}

Fuente: elaboración propia

La media de número de publicaciones por año en todas las revistas -teniendo en cuenta las fluctuaciones de producción científica- ronda los 40 artículos por año y por revista, con un visible pico de un 30\% en los años 2013 y 2014 (71 y 62, respectivamente), que se debe a la mayor producción de las revistas arriba mencionadas.

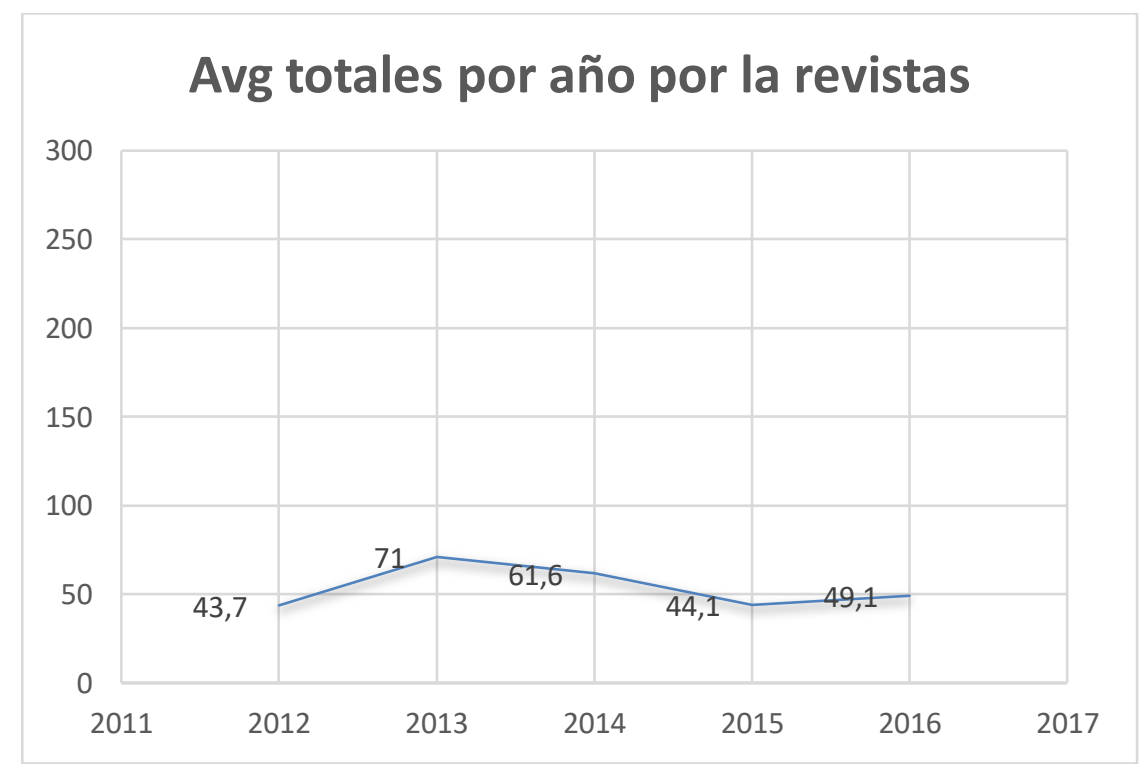

Gráfico 3. Media de producción por año dentro del periodo analizado

Fuente: elaboración propia

Sin embargo, la evaluación anual demuestra variaciones considerables, en cuanto al número de las publicaciones en diferentes revistas y diferentes años. Como se ha indicado antes, Comunicar sigue siendo la única revista que publica el mismo número de artículos cada año -200 en total entre 2012 y 2016-, seguido por Comunicación y Sociedad, El Profesional de Información y SCIRE, con parecida política de estabilidad en el volumen de publicaciones de un número a otro. Signa e Ibersid han doblado el número de publicaciones y Revista Latina de Comunicación Social lo ha cuadriplicado. 
En cuanto a las variaciones anuales, 2012 y 2016 son los años con menores diferencias 0 , más bien, con diferencias menos abruptas entre revistas. El periodo entre 2013 y 2015 son años en los que hay más variaciones entre revistas. Destaca el caso de Historia y Comunicación Social, que ha incrementado su volumen de publicaciones de 18 en 2012 a más de 200 en los años 2013 y 2014, para bajarlo en los años posteriores. Otro ejemplo de cambios de semejante naturaleza es el de Estudios sobre el Mensaje Periodístico.

De este modo, se puede destacar que, a pesar de gran volumen de publicaciones anuales en su totalidad y de media, por año y por revista, cuatro revistas destacan la estabilidad editorial en cuanto al número de artículos publicados en cada año, tres revistas han aumentado el volumen de publicaciones y dos demuestran gran variedad de un año a otro y de un número a otro. El análisis anual por revistas también demuestra la variedad en el número de artículos publicados.

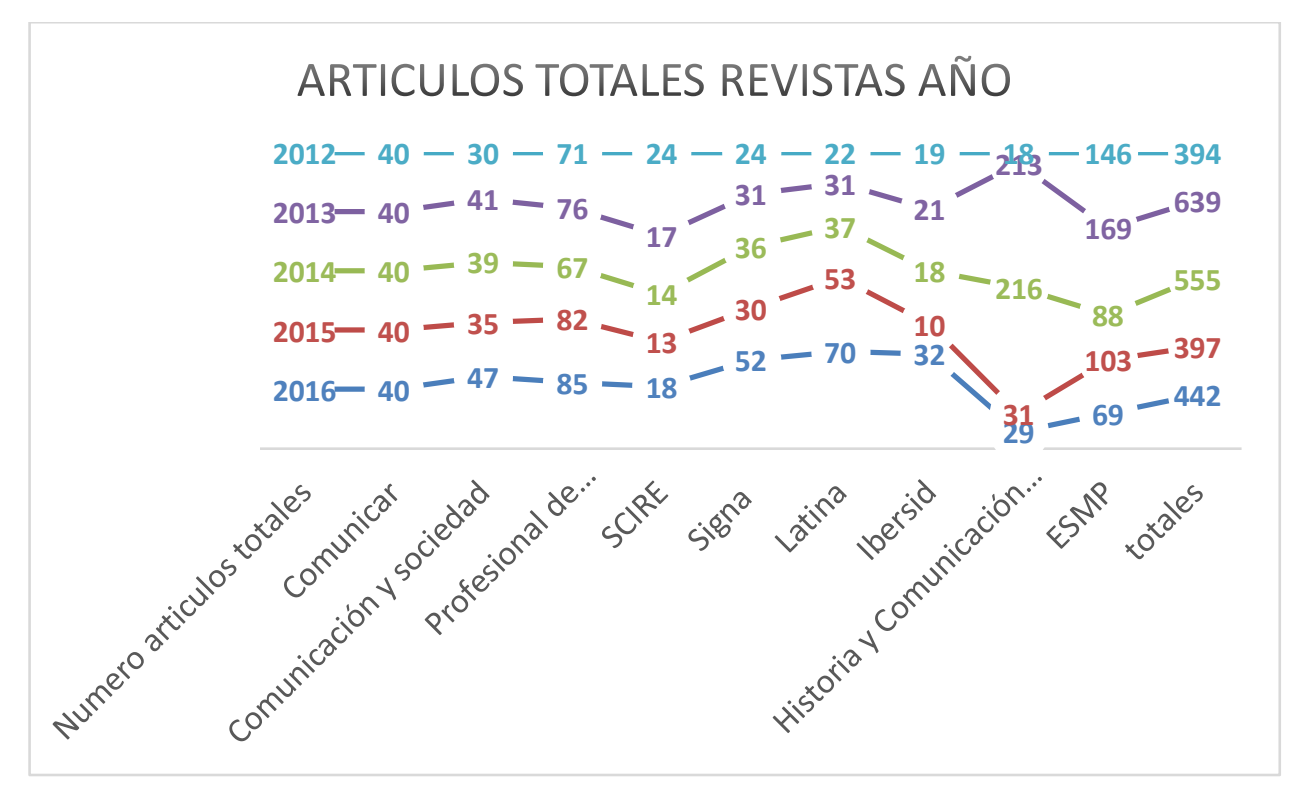

Gráfico 4. Variaciones del volumen de publicaciones por año y revista Fuente: elaboración propia

El año 2013 y 2014 se registraron cifras record en cuanto al número de publicaciones, sobrepasando en un $30 \%$ la media anual del volumen total de publicaciones en el periodo analizado: concretamente, fueron 639 publicaciones en 2013 y 555 en 2014.

Centrándonos en las publicaciones sobre Social Media, se ha comprobado que, entre los 2427 artículos publicados en las revistas de impacto del campo de las Ciencias de Comunicación españolas en los últimos 5 años, 316 están dedicadas a esta temática, lo que constituye 13\% de los artículos publicados desde 2012 hasta 2016. 


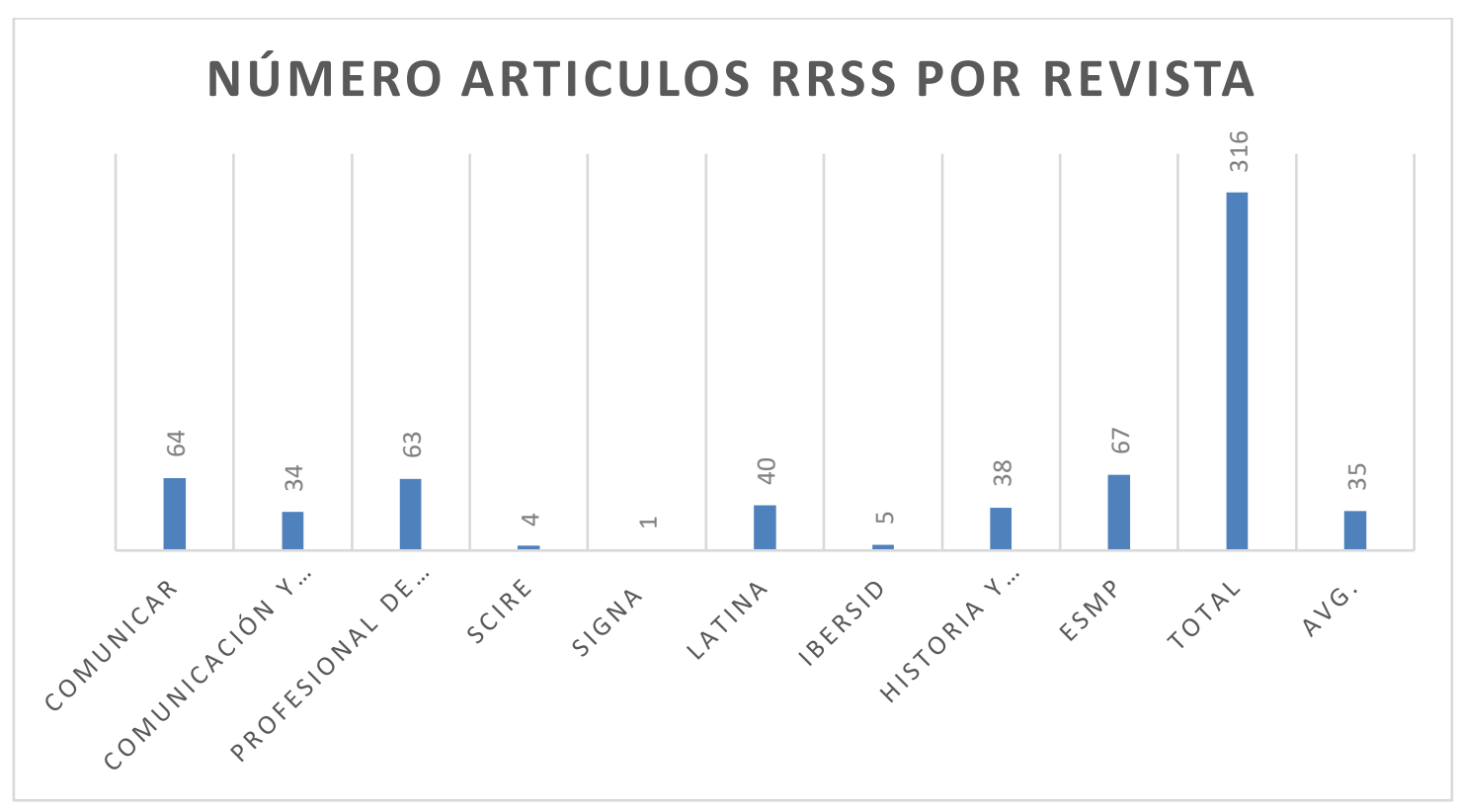

Gráfico 5. Número de artículos sobre redes sociales en cada revista

Fuente: elaboración propia

El gráfico 4 muestra cómo, de los 316 artículos sobre la temática en cuestión, las revistas que más han publicado han sido Estudios sobre el Mensaje Periodístico (67), Comunicar (64) y El Profesional de Información (63). Son las tres revistas en las que los Social Media ocupan un espacio considerable como eje temático, destacando por la innovación en investigación y publicación sobre los temas emergentes. En el extremo contrario se sitúan Ibersid, con 5 artículos, Scire, con 4, y Signa, con 1. En el período analizado, se ve claramente que estas revistas tienen un perfil investigador que no están centrado en la Comunicación digital como tal, sino que el área está presente de forma marginal e, incluso, está ausente en muchos números.

La media de artículos sobre Social Media publicados por revista en los 5 años analizados es de 35 , frente a los 270 artículos que publica de media cada revista es este período. 


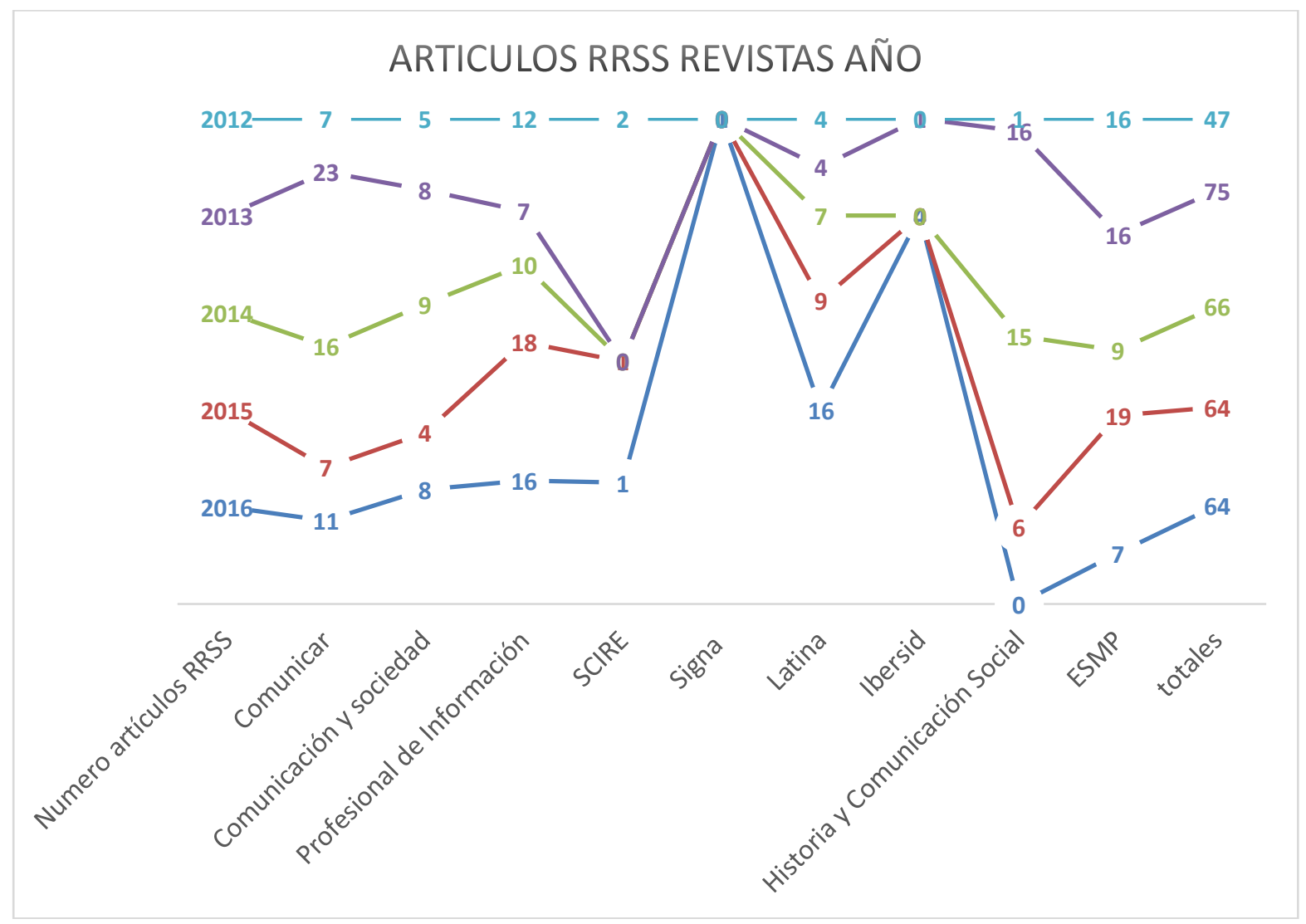

Gráfico 6. Variaciones del volumen de publicaciones por año y revista de los artículos sobre las redes sociales

Fuente: elaboración propia

El análisis de variación de publicación sobre redes sociales por revista y por año aporta una perspectiva muy detallada del desarrollo del interés por esta temática desde 2012 hasta actualidad en las diferentes revistas de impacto. Se observa que la tendencia general de publicación sobre Social Media experimenta un claro crecimiento y, a su vez, cierta estabilidad, con tendencia a bajar (en cuanto al número total de publicaciones sobre redes sociales en el sector de revistas de alto impacto); a pesar de la creciente complejidad del área de Social Media y del interés profesional y académico que ha conseguido este ámbito de la Comunicación.

Esta tendencia se muestra contraria a los picos y bajadas observados en la producción científica general en estas revistas. Desde 2012, cuando hubo 47 publicaciones, el número ha ido creciendo hasta más de 60 artículos por año. El número que se mantiene casi estable desde 2014. Destaca, con un pico de 75 publicaciones el año 2013 que se debe, en gran medida, a la publicación de 23 artículos en Comunicar. Asimismo, en 2015, Estudios sobre el Mensaje Periodístico hace la gran aportación de 19 artículos publicados sobre la temática. El año 2013, en general, con la aportación de Comunicar y el caso de Estudios sobre el Mensaje Periodístico en 2015, son los momentos en los que se detecta el mayor interés científico en el eje temático de las herramientas digitales. Sería interesante profundizar en esta dirección para descubrir los motivos que justifican este fenómeno. 
En relación a cada revista, se observa que Comunicar registró el mayor crecimiento en 2013 y 2014 (23 y 16, respectivamente), mientras que los años anteriores y posteriores muestran menor nivel de interés en este aspecto y también en comparación con otras revistas en el marco anual.

Por su parte, Comunicación y Sociedad -salvo en los años 2012 y 2015, años en los que publica menos- mantiene estable el número de artículos: alrededor de 10 publicaciones al año sobre los medios sociales digitales.

Semejante situación se puede apreciar en Estudios sobre el Mensaje Periodístico, que también mantiene un elevado número de publicaciones sobre este tema en 3 años distintos: 2012, 2013 y 2015. Es llamativo este pico de interés y de publicaciones abundantes durante estos años; y que disminuya a la mitad el número de publicaciones en 2016.

La clara tendencia a publicar menos sobre redes sociales durante los últimos años se constata también en Historia y Comunicación Social. La revista registró dos picos, en 2013 y 2014 (con 16 y 15 artículos publicados sobre esta temática, respectivamente), mientras son casi inexistentes los artículos sobre el tema en 2012 y 2016.

El Profesional de Información destaca por su enfoque innovador, con 12 publicaciones en 2012, al inicio del boom de las redes sociales, junto con Estudios sobre el Mensaje Periodístico, con 16 títulos ese mismo año. Sin embargo, El Profesional de la Información demuestra una tendencia creciente del interés editorial y de la investigación reflejada en las publicaciones en cuanto a las redes sociales, junto con Revista Latina de Comunicación Social. En el caso de esta última revista, el crecimiento del número de publicaciones sobre redes sociales es aún más marcado.

En general, podemos observar que los Social Media registran picos de interés, con sus correspondientes caídas o descensos en cuanto al número de publicaciones. Solo en el caso de dos revistas podemos hablar de una tendencia de crecimiento claro de la investigación en esta área (El Profesional de la Información y Revista Latina de Comunicación Social) o cierta estabilidad (Comunicar) durante el periodo investigado. Aunque observamos que en los últimos años los medios digitales protagonizan más la investigación que en el año 2012, los años 2013-2015 fueron los que supusieron un mayor número de publicaciones en esta temática. 


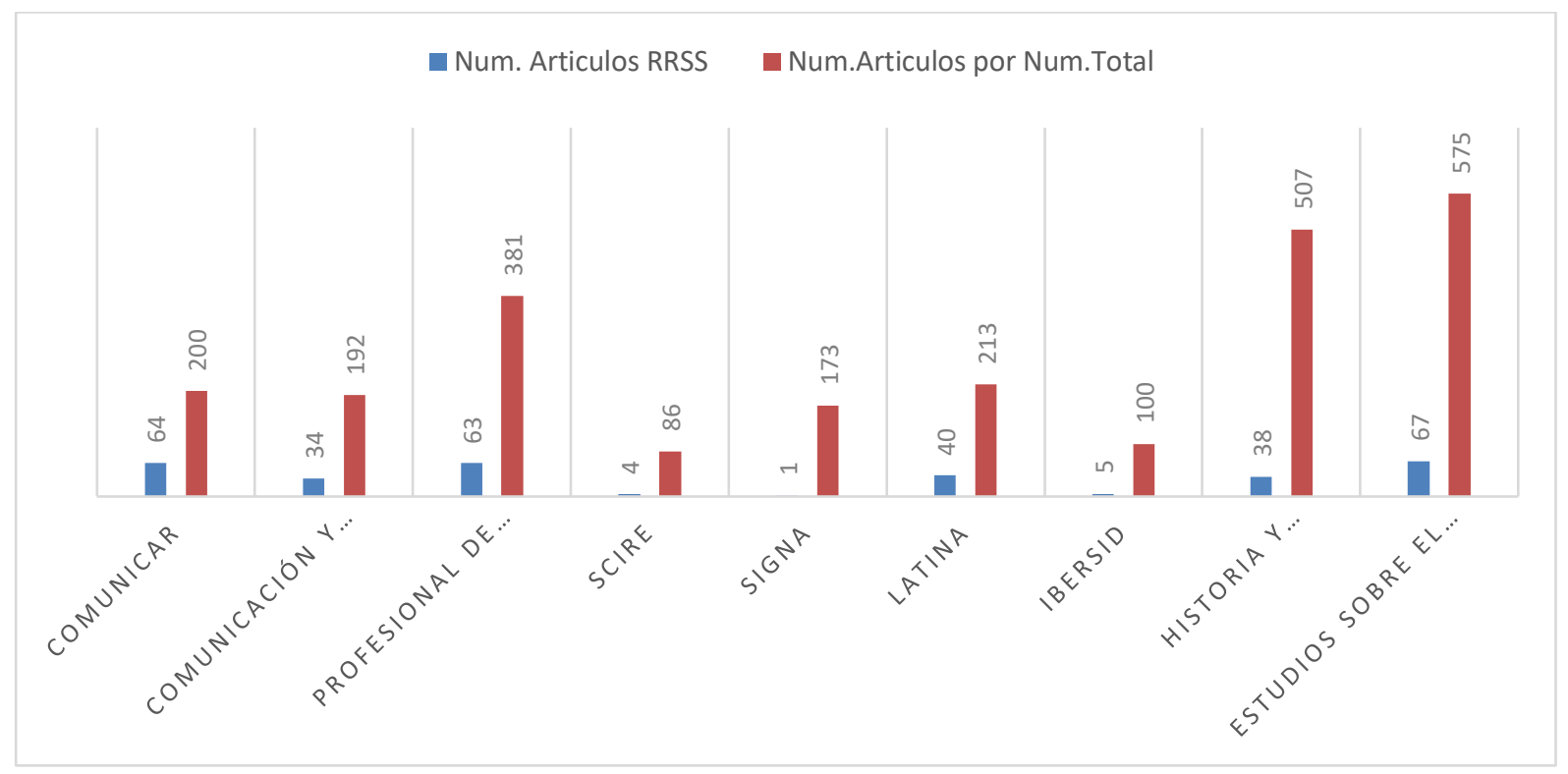

Gráfico 7. Comparación entre el número total de publicaciones y sobre redes sociales por revista

Fuente: elaboración propia

En general, los artículos sobre redes sociales fueron escasos, si tomamos como referencia el número total de publicaciones entre 2012 y 2016. Las revistas con mayor número de publicaciones sobre la temática objeto de estudio, respecto al total, fue Estudios sobre el Mensaje Periodístico (con 67) y, en segundo lugar, Comunicar (con 64); seguido en tercer lugar por El Profesional de la Información (con 63). De tal modo que las dos revistas con mayor número de publicaciones en el periodo analizado son también las que más publican sobre Social Media: Estudios sobre el Mensaje Periodístico y El Profesional de la Información. La excepción la revista Historia y Comunicación Social, en la que esta relación no se da. Comunicar y Revista Latina de Comunicación Social, por ejemplo, suponen el mayor porcentaje de publicaciones acerca de redes sociales. Concretamente, se han hallado 64 artículos sobre redes sociales, entre los 200 publicados por Comunicar; y 40 de 213, en el caso de Revista Latina de Comunicación Social. 


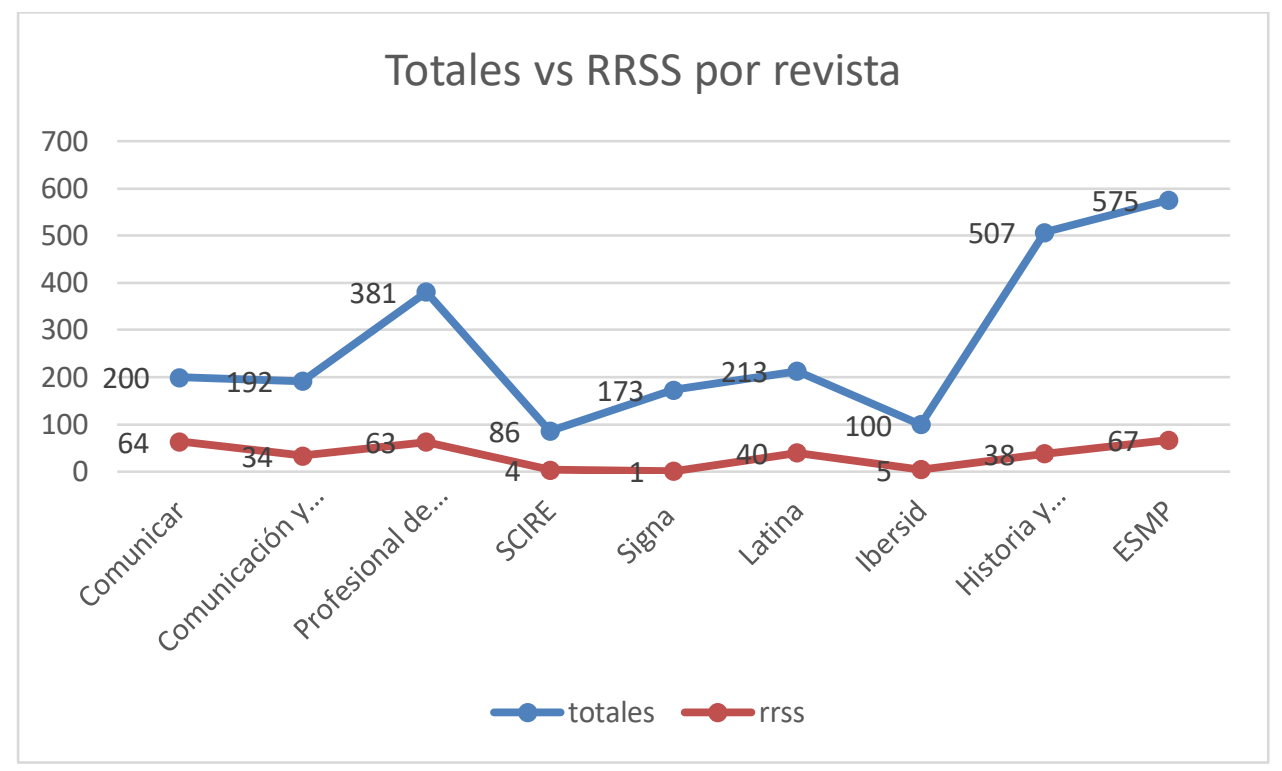

Gráfico 8. Comparación entre publicaciones totales y sobre redes sociales, por revista, en el período 2012-2016

Fuente: elaboración propia

Esta relación demuestra que el equilibro entre número de publicaciones y elevado interés en el campo de medios digitales es, indudablemente, más útil en cuanto al impacto. Sin embargo, es noticiable que, en general, la investigación dedicada a este tema emergente es relativamente bajo, en el caso de todas las revistas en el periodo analizado (tal como lo demuestra la Figura 7). Es bajo a pesar de registrar picos, tanto en el crecimiento en producción científica en general en Comunicación como en esta área específica, estando relacionados entre sí (véase la Fig.8).

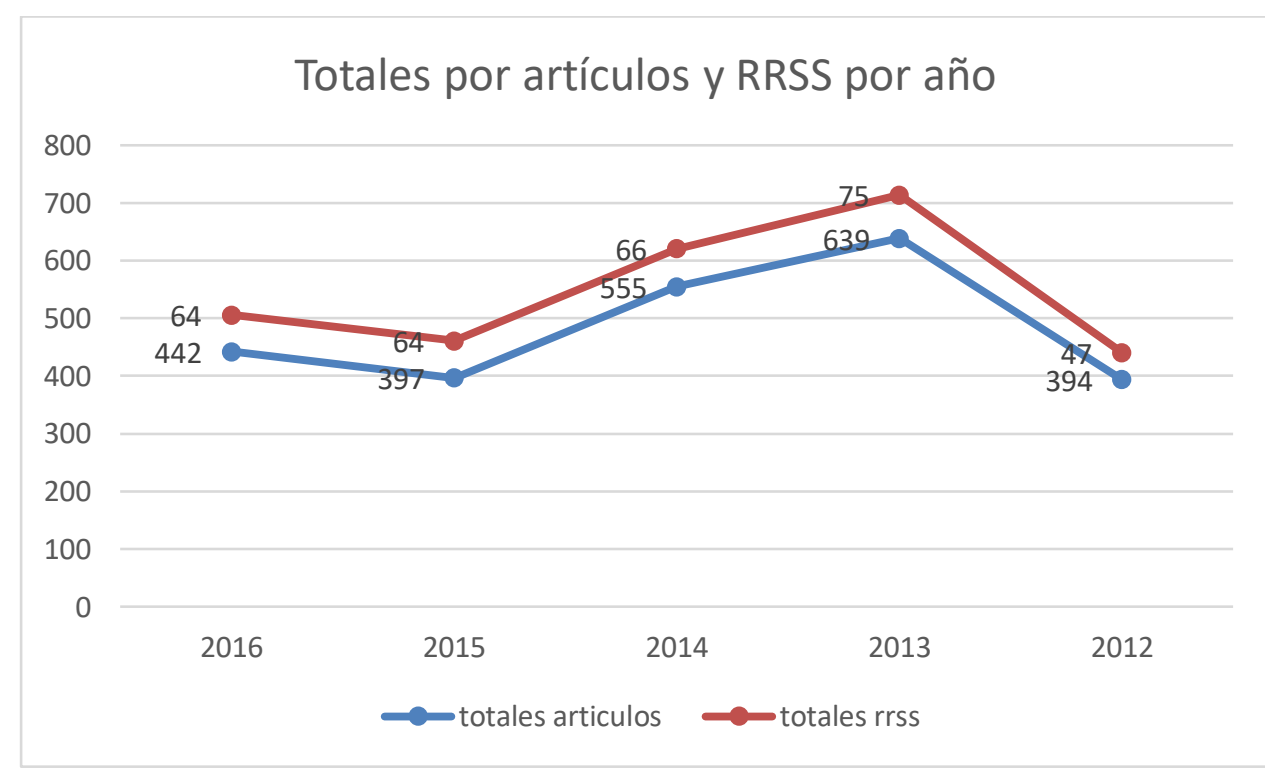

Gráfico 9. Comparación entre publicaciones totales y sobre las redes sociales, por revista, en el período 2012-2016

Fuente: elaboración propia 
Los números nos dan una idea general sobre la perspectiva de la investigación, tanto en producción científica total en los años analizados como por revistas. Del mismo modo, en el caso de las publicaciones sobre medios sociales digitales. Por lo tanto, es de suma importancia ver estas comparaciones.
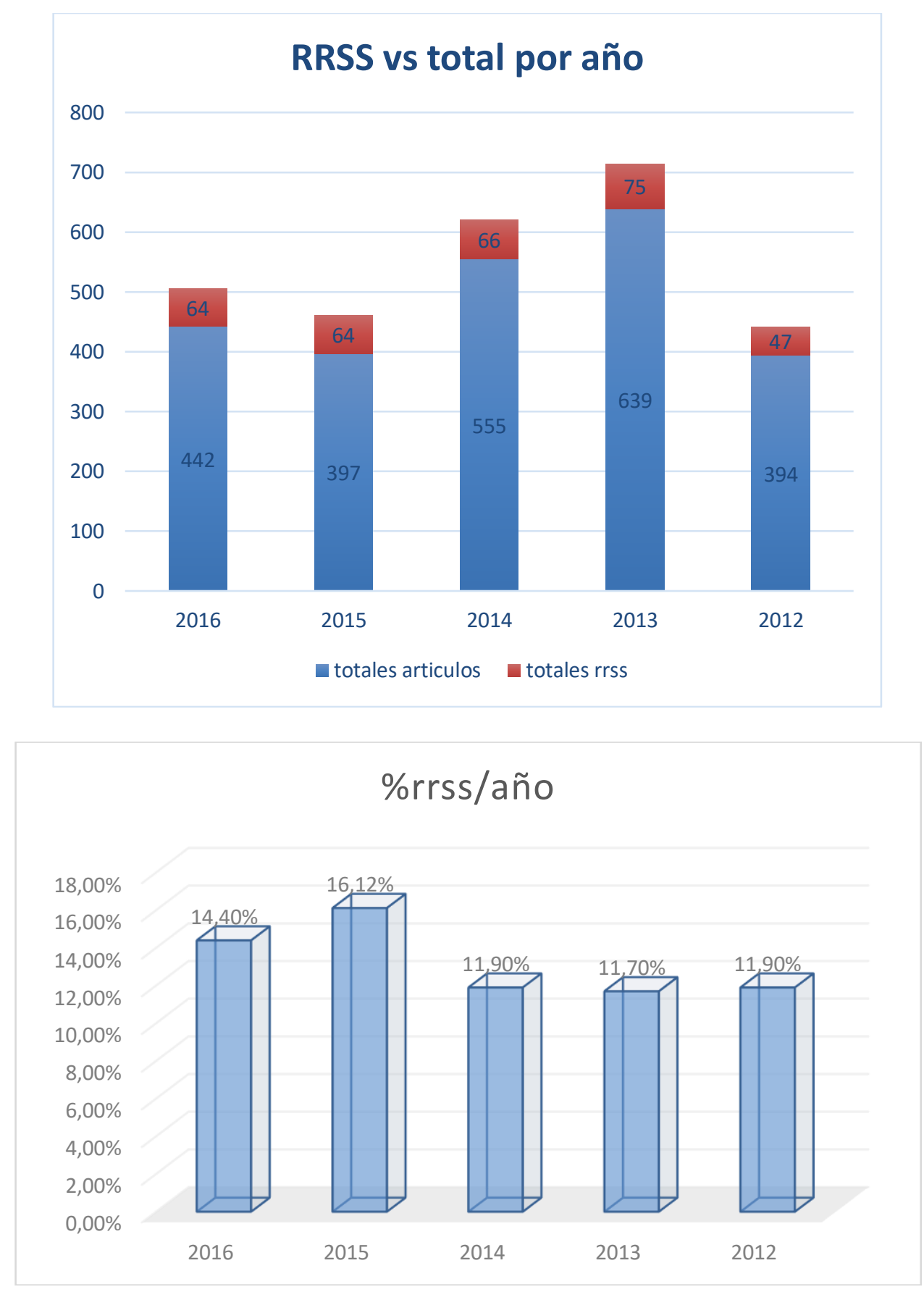

Gráfico 10. Producción científica total y sobre redes sociales, en números reales y porcentajes

Fuente: elaboración propia

Los años 2013 y 2014, seguido por año 2016, fue el periodo con mayor número de publicaciones sobre redes sociales en las revistas de impacto, aunque no alcanzan ni las 100 publicaciones por año. Sin embargo, si relacionamos los números, resulta que, 
a pesar del crecimiento de publicaciones observado en estos años, 2015 cuenta con mayor porcentaje de investigación de impacto que verse sobre los Social Media, seguido por el año 2016. De este modo, los Social Media entran con más impulso a la investigación académica de impacto recientemente, a pesar de los picos registrados en años anteriores.

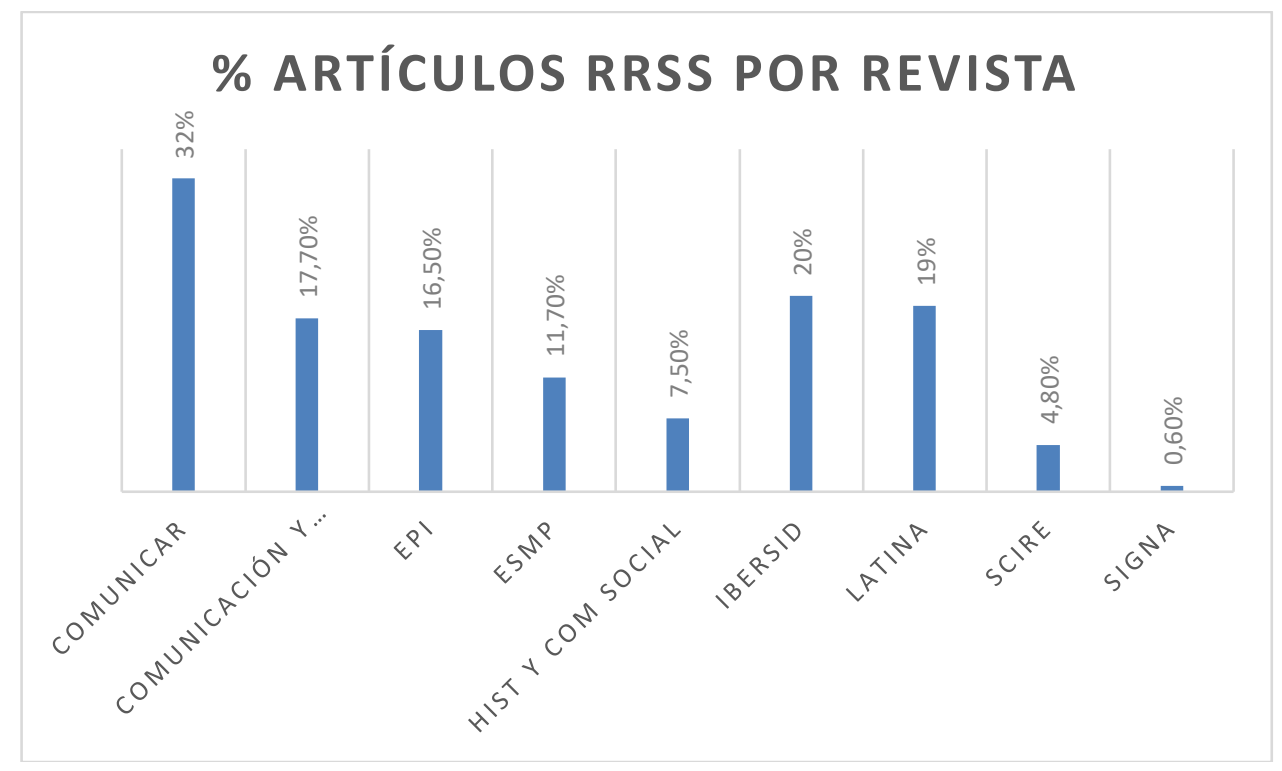

Gráfico 11. Porcentaje de los artículos sobre las redes sociales, por revista, en el período 2012-2016

Fuente: elaboración propia

Tras identificar las revistas con mayor número de publicaciones en general y, especialmente, sobre redes sociales, en el campo de la Comunicación, podemos ver que las revistas de investigación académica de calidad en los últimos 5 años con un perfil claro en Social Media son: Comunicar (con un $32 \%$ de sus artículos sobre redes sociales), Revista Latina de Comunicación Social (con un 19\%), Comunicación y Sociedad (17.7\%) y El Profesional de la Información (16.5\%). De esta vanguardia de investigación en Comunicación de impacto excluimos a lbersid porque, a pesar de contar con $20 \%$ de las publicaciones sobre el tema en cuestión, cuenta en general con un pequeño número de publicaciones en comparación con otras revistas, resultando relativamente insignificante en cuanto al número total de artículos publicados por otras revistas.

En cuanto a los artículos sobre redes sociales, se reparten prácticamente entre 4 revistas: Comunicar (28\%), Comunicación y Sociedad (15\%), Revista Latina de Comunicación Social (17\%) y El Profesional de la Información (14\%). Son las pioneras en el período analizado en la investigación de calidad sobre los medios sociales digitales, destacando su enfoque innovador en este sentido. 
ESTUDIO DE LA PRODUCCIÓN CIENTÍFICA SOBRE SOCIAL MEDIA. EL CASO DE LAS REVISTAS ESPAÑOLAS DE COMUNICACIÓN EN JCR Y SJR

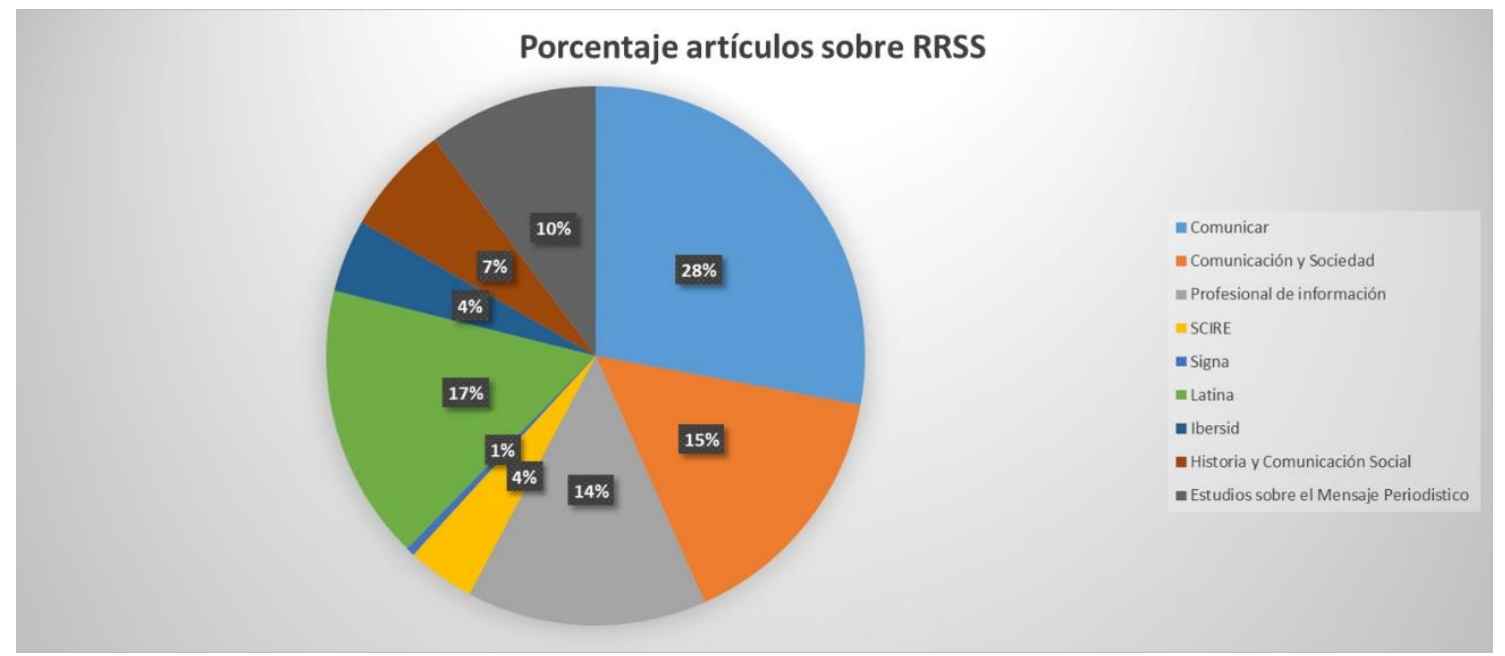

Gráfico 12. Distribución de los artículos sobre redes sociales en las revistas con impacto en el periodo 2012-2016

Fuente: elaboración propia

Para ilustrar definitivamente el peso que tiene cada revista en la producción científica sobre el nuevo campo de la Comunicación digital y redes sociales, hemos analizado las medias del número de artículos dedicados a la temática en cuestión por año y por revista. 2013 es, otra vez, el año con mayor dedicación a la temática de redes sociales, con la posterior estabilidad en los años más recientes, aunque son los últimos años los que registran más publicaciones -sobre todo el 2015 y 2016. La media por año se puede comprobar en la Fig. 12.

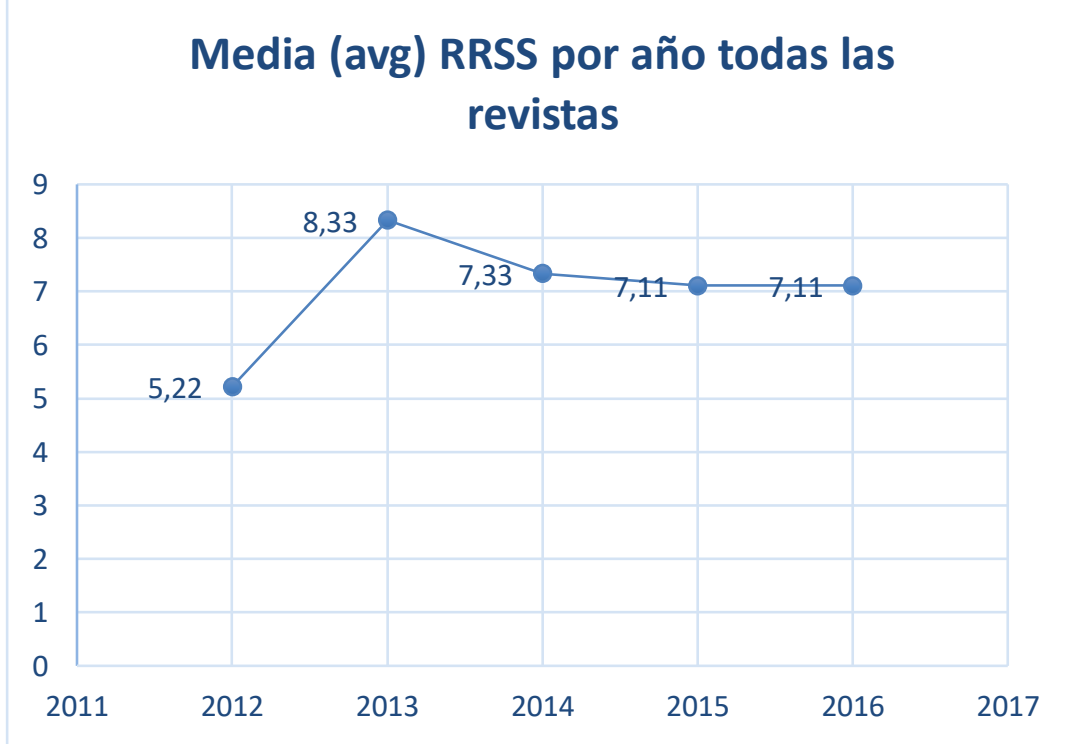

Gráfico 13. Media de publicaciones sobre redes sociales, por año, en el periodo analizado

Fuente: elaboración propia

Si comparamos revistas, los primeros lugares los ocupan Estudios sobre el Mensaje Periodístico, Comunicary El Profesional de la Información. Las medias de los artículos 
centrados en redes sociales suelen coincidir con las medias de producción científica general, aunque aquí hay que destacar la revista Historia y Comunicación Social y, en segundo lugar, Estudios sobre el Mensaje Periodístico. Pero no siempre se va a demostrar esta tendencia tan evidente. Si miramos las medias más estables de publicaciones en su totalidad y las comparamos con las medias de artículos sobre redes sociales, Comunicar y El Profesional de la Información son las dos que en el periodo analizado demuestran, de manera destacada, esta perspectiva de aportar el enfoque académico a la temática emergente, seguido por la Revista Latina de Comunicación Social. En este sentido, se pueden considerar líderes entre 2012 y 2016, mientras otras revistas demuestran variaciones con ascensos y descensos 0 suponen una relación casi insignificante.

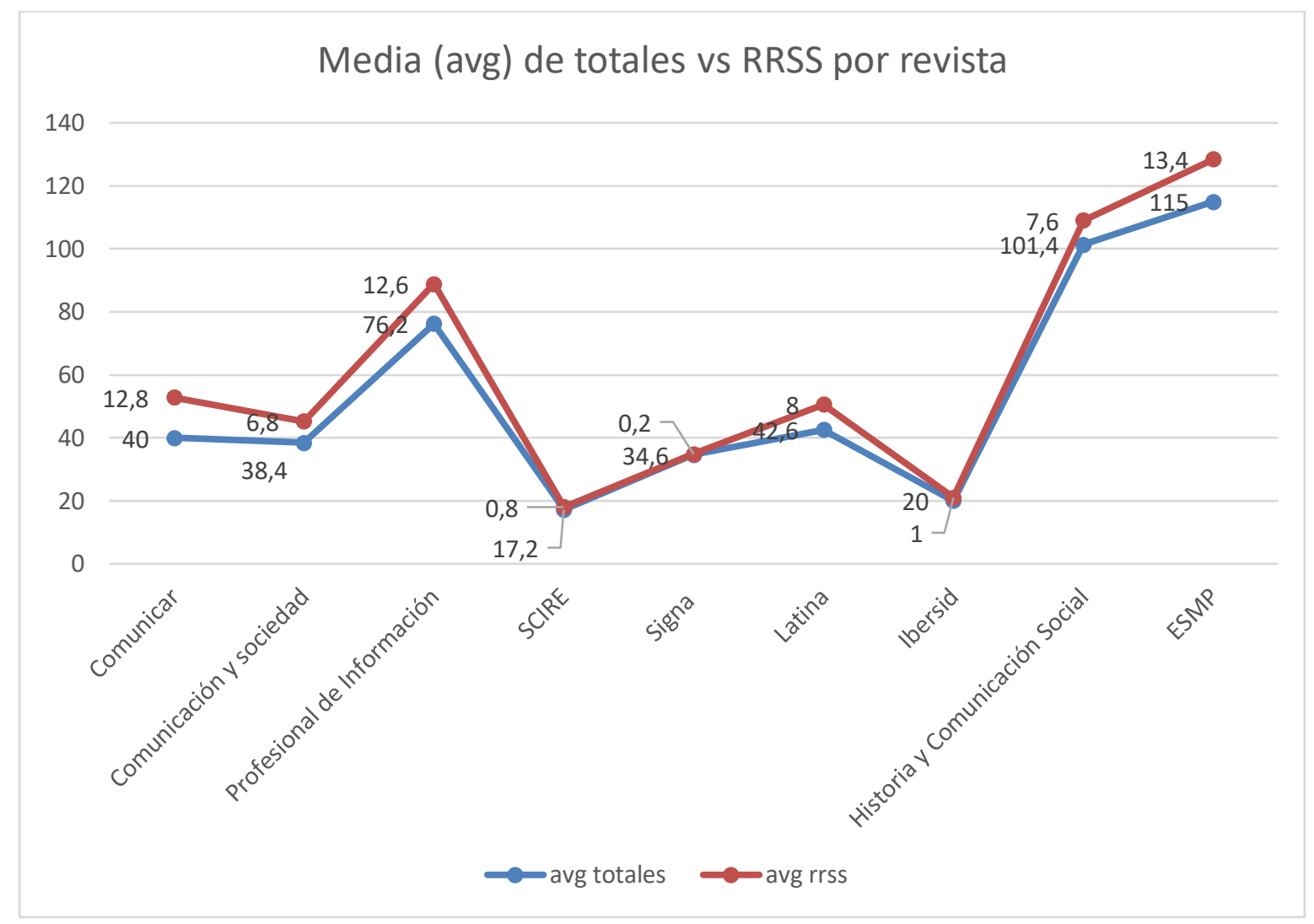

Gráfico 14. Media de publicaciones sobre redes sociales, por la revista, en el período analizado

Fuente: elaboración propia

\section{CONCLUSIONES}

El análisis de los resultados demuestra que es necesario abordar el estudio de los datos desde distintos procedimientos y perspectivas, relacionando los números y datos tanto por años como por las revistas, para poder obtener una radiografía más detallada y profunda. De este modo, nos parece oportuno el método aplicado, que se basa en los procedimientos estadísticos descriptivos desde diferentes enfoques: porcentajes, medias, comparaciones, curvas de desarrollo, etc. Este amplio procedimiento de análisis permite llegar a conclusiones fundamentadas y justificadas. 
De tal modo, primero debemos considerar que, en general, las revistas españolas indexadas en dos de los principales índices de revistas en el campo de las Ciencias de Comunicación, de 2012 a 2016 dedican un espacio relativamente pequeño a la vanguardista temática de las redes sociales: un $13 \%$ de las publicaciones. A pesar del gran número de publicaciones (2427 en total en el periodo analizado), las vinculadas a redes sociales suponen apenas el $13 \%$. Esto nos indica que la temática, a pesar del desarrollo y asentamiento en la práctica diaria en el mundo profesional, además del creciente interés docente y profesional, no consigue una dedicación notable en cuanto a la investigación de prestigio.

En segundo lugar, a pesar de altas medias de publicaciones en general por año (485) o por revistas (242) en el período analizado, la media de publicaciones sobre redes sociales es relativamente baja y ronda 13 artículos por revista y 7.33 por año. Sería interesante también investigar los motivos de las fluctuaciones observada en cuanto al número de publicaciones en el año 2014 y 2016.

Teniendo en cuenta que la preparación universitaria requiere, cada vez más, dedicar atención a los asuntos vinculados a los medios digitales, la investigación académica debería acompañar al mundo profesional y docente con proyectos de investigación de calidad sobre esta temática. En general, aquí se detectan aspectos mejorables en cuanto a la labor que han venido desarrollando los científicos de la Comunicación. El fenómeno de los Social Media ya se ha asentado, las prácticas humanas y empresariales están generalizadas y, en consecuencia, el alcance de su impacto debe ser investigado desde ámbitos científicos, para lograr entenderlo mejor.

Además, cabe destacar la gran variación anual, en cuanto al número total de publicaciones y artículos dedicados a la temática en cuestión. Destaca el gran número de publicaciones en 2013 y 2014, sobre todo, o en revistas como Estudios sobre el Mensaje Periodístico o Historia y Comunicación Social. Comunicar es la única revista con una estable política de publicaciones, y la Revista Latina de Comunicación Social destaca por su crecimiento, considerable pero razonable, del número de publicaciones. El gran volumen de publicaciones, en definitiva, por año y por revista, refleja el tremendo impacto que tienen las revistas incluidas en los mejores índices sobre el campo de Comunicación. Sin embargo, unas políticas editoriales, que establezcan los criterios claros del volumen de producción científica, serían de gran ayuda para hacer este sector más transparente.

Estas variaciones anuales son aún más evidentes en el caso de las revistas y los años concretos, en cuanto a los artículos dedicados a la temática de los Social Media. A pesar de los picos observados, el desarrollo anual del número de publicaciones sobre este tema indica un estable crecimiento en los últimos años, más de 60 artículos por año. Esto se confirmó con un análisis porcentual, con el que se demostró que los años 2015 y 2016 registran mayor interés en la temática a medida que se desarrollan las redes sociales (a partir de 2012). La estabilidad de la media de publicaciones sobre redes sociales en los últimos 3 años en todas las revistas lo justifica. Las fluctuaciones son aún más evidentes en el caso de las revistas en los distintos años, en los que Comunicar, Revista Latina de Comunicación Social y El Profesional de la Información demuestran cierta estabilidad o crecimiento en cuanto a la producción científica versada en redes sociales. 
Por su parte, la variación de media de publicaciones totales indica la necesidad de trabajar las políticas editoriales en revistas prestigiosas en el campo de la Comunicación., salvo en casos excepcionales como Comunicar, El Profesional de la Información o Revista Latina de Comunicación Social, que mantienen políticas estables en cuanto al número de artículos a publicar en cada volumen.

A este panorama debemos añadir que la elevada publicación de artículos en las revistas de impacto, acompañada en varios casos por el elevado número de publicaciones dedicadas al campo de vanguardia de la Comunicación, no se relaciona claramente con el perfil líder o innovador de la revista en este campo. Los resultados manifiestan que las tres revistas (Comunicar, Revista Latina de Comunicación Social y El Profesional de la Información) demuestran una clara orientación a la investigación en la temática comunicativa emergente en los últimos años y son las revistas incluidas con los mejores índices de impacto. Junto con Comunicación y Sociedad, son responsables del $75 \%$ de las publicaciones sobre redes sociales en los últimos cinco años.

Sin embargo, el gran volumen de producción científica no va acompañado de un elevado interés en los Social Media en las revistas indexadas en JCR y SJR, aunque se suponía lo contrario. Las variaciones entre la producción científica total y la dedicada a los Social Media, tanto desde la perspectiva anual como por revista, también indica la necesidad de estabilizar y mejorar en este punto. Aunque los últimos años demuestran el crecimiento y estabilidad de la investigación de calidad en Social Media, se necesitan mayores esfuerzos de las políticas editoriales y la motivación intrínseca y extrínseca de los investigadores en ciencias de Comunicación hispanohablantes.

Asimismo, el análisis desde varios enfoques nos ha permitido identificar cuáles son las revistas de impacto con la mayor orientación hacia la innovación comunicativa; siendo, de este modo, las que ostentan el liderazgo entre las revistas indexadas y en el campo científico en general

En cuanto a las futuras líneas de investigación, cabe destacar que, aunque el estudio multi-variable y multi-enfoque nos ha permitido establecer ciertas pautas e indicar los puntos de interés, se precisa el desarrollo de estudios en el futuro próximo que aborden la producción científica de impacto sobre redes sociales y Comunicación; para poder profundizar en el conjunto de los artículos identificados en cuanto a su orientación metodológica, temática y campo de aplicación, así como datos científicos de autores, centros y proyectos. Este estudio se plantea en un futuro próximo, acompañado por la investigación, más a largo plazo, que indague en las razones de los picos observados; así como las motivaciones de los investigadores en cuanto a su limitado interés científico en el tema en cuestión.

\section{REFERENCIAS}

Almansa-Martínez, A. y Castillo-Esparcia, A. (2014). Comunicación Institucional en España. Estudio del uso que los diputados españoles hacen de las TIC en sus 
relaciones con la ciudadanía. Chasqui: Revista Latinoamericana de Comunicación, $126,22-30$.

Almansa-Martínez, A., Fonseca, Ó. y Castillo-Esparcia, A. (2013). Redes sociales y jóvenes.: Uso de Facebook en la juventud colombiana y española. Comunicar, (40), 127-134.

Barón Pulido, M., Duque Soto, Á., Mendoza Lozano, F., \& Quintero Peña, W. (2021). Redes sociales y relaciones digitales, una comunicación que supera el cara a cara. Revista Internacional De Pedagogía E Innovación Educativa, 1(1), 123-148. https://doi.org/10.51660/ripie.v1i1.29

Boyd, D. y Ellison, N. (2008). Social Network Sites: Definition, History, and Scholarship. Journal of Computer-Mediated Communication, (13), 210-230.

Caldevilla Domínguez, D. (2010). Las Redes Sociales. Tipología, uso y consumo de las redes 2.0 en la sociedad digital actual. Documentación de las Ciencias de la Información, Vol. 33, 45-68. https://revistas.ucm.es/index.php/DCIN/article/view/DCIN1010110045A/18656

Canaza-Choque, F. A. (2018). La sociedad 2.0 y el espejismo de las redes sociales en la modernidad líquida. In Crescendo, vol. 9, núm. 2, 221-247. https://www.aacademica.org/franklin.americo.canazachoque/6

Castillo-Esparcia, A. y Carretón-Ballester, M. C. (2010). Investigación en Comunicación. Estudio bibliométrico de las Revistas de Comunicación en España. $\begin{array}{lllll}\text { Comunicación } y & \text { (23)2, } & \text { 289-327. }\end{array}$ https://rua.ua.es/dspace/bitstream/10045/22678/1/2010 Comunicacion y Socied $\underline{\text { ad.pdf }}$

Castillo-Esparcia, A., Almansa-Martínez, A. y Smolak-Lozano, E. (2015). East European think tanks in social media - towards the model of evaluation of effective communication/PR strategies: Case study analysis. Catalan journal of communication \& cultural studies, (7)2, 231-250

Castillo-Esparcia, A., Rubio-Moraga, Á. y Almansa-Martínez, A. (2012). La Investigación en comunicación. Análisis bibliométrico de las revistas de mayor impacto del ISI. Revista Latina de Comunicación Social, (67), 248- 270.

Codina, L. (2018). Revisiones bibliográficas sistematizadas: procedimientos generales y Framework para ciencias humanas y sociales. Barcelona: Universitat Pompeu Fabra, Departamento de Comunicación, Máster Universitario en Comunicación Social, p. 87

De Filippo, D. (2013). La producción científica española en Comunicación en WOS: Las revistas indexadas en SSCI (2007-2012). Comunicar, (41), 25-34.

De Pablos-Coello, J. M., Túñez-López, J. M. y Mateos-Martín, C. (2015). El idioma, como vehículo de la comunicación científica: análisis de la visibilidad e impacto de 
la investigación a través de la bibliometría de Google. Estudios sobre el Mensaje Periodístico, (21) 1, 283-298.

Diga, M. y Kelleher, T. (2009). Social media use, perceptions of decision-making power, and public relations roles. Public Relations Review, 35(4), 440-442.

Estudio Anual de Redes Sociales 2019. Madrid: IAB Spain y Elogia, 2019

Eyrich, N., Padman, M., Sweetser, K. (2008). PR practitioners' use of social media tools and communication technology. Public Relations Review, (34), 412-414.

Giménez-Toledo, E. (2013). Reflexiones sobre las revistas científicas españolas. Anuario Think EPI, (1), 118-121.

Hernández Salazar, P. (2019). Metodología cualitativa en bibliotecología y ciencia de la información. Un análisis bibliográfico de artículos académicos". Investigación Bibliotecológica: archivonomía, bibliotecología e información. 33 (78), 105-120. http://dx.doi.org/10.22201/iibi.24488321xe.2019.78.58024

Landeta Bejarano, Z., Salamea Limones, V., \& Montecé Mosquera, F. (2020). Redes Sociales y Periodismo Ciudadano: Investigación Documental. Revista Ciencia E $\begin{array}{lllll}\text { Investigación. } & 5(1), & 149 & - & 164 .\end{array}$ https://revistas.utb.edu.ec/index.php/sr/article/view/761

Leydesdorff, L. y Probst, C. (2009). The Delineation of an Interdisciplinary Specialty in Terms of a Journal Set: The Case of Communication Studies. Journal of the American Society for Information Science and Technology, 60(8).

Martínez-Nicolás, M. y Saperas-Lapiedra, E. (2011). La investigación sobre Comunicación en España (1998-2007). Análisis de los artículos publicados en revistas científicas. Revista Latina de Comunicación Social, 66, 101-129.

Montero-Díaz, J., Cobo, M. J., Gutiérrez-Salcedo, M., Segado-Boj, F. y HerreraViedma, E. (2018). Mapeo científico de la Categoría «Comunicación» en WoS (1980-2013). Comunicar, (55), 81-91.

Park, H., Leydesdorff, L. (2009). Knowledge linkage structures in communication studies using citation analysis among communication journals. Scientometrics, 81(1), 157-175.

Ramos Soler, I., Del-Pino-Romero, C. y Castello-Martínez, A. (2014). Web 2.0 y redes sociales: estudio de las publicaciones científicas en las revistas españolas de comunicación. Historia y comunicación social, (19)1, 577-590.

Repiso-Caballero, R. y Torres-Salinas, D. (2014). Comunicación y documentación: dos disciplinas convergentes pero distintas. Anuario Think EPI, (1), 225-229.

Repiso, R., Torres-Salinas, D. y Delgado López-Cózar, E. (2016). Análisis de la relación entre disciplinas a través del uso de tesis doctorales. El caso de Televisión, 
Radio, Cine y Fotografía en España. Revista Latina de Comunicación Social, (71), 874-890.

Rosero Molina, J., Rodríguez Chaparro, D. y Ramos Maldonado, C. (2020). Sistematización de los artículos de grado de las especializaciones en comunicación de la UAC. Revista de Ciencias de la Comunicación e Información, 25(3), 57-70. http://doi.org/10.35742/rcci.2020.25(3).57-70

Rúas Araújo, J. y Casero-Ripollés, A. (2018). Comunicación política en la época de las redes sociales: lo viejo y lo nuevo, y más allá. adComunica: revista científica de estrategias, tendencias e innovación en comunicación, 16, 21-24.

Sánchez-Hervás, D. (2013). Estrategias de comunicación en redes sociales. Usuarios, aplicaciones y contenidos. Sphera pública: revista de ciencias sociales y de la comunicación, (1)13, 183-185.

Torres-Salinas, D., Delgado-López-Cózar, E. y García-Moreno-Torres, J., Herrera, F. (2011). Rankings ISI de las universidades españolas según campos científicos: descripción y resultados. El Profesional de la Información, (20)1, 111-118.

Torres-Salinas, D., Jiménez-Contreras, E. y Robinson-García, N. (2014). Tendencias en mapas de la ciencia: co-uso de información científica como reflejo de los intereses de los investigadores. El Profesional de la Información, (23)3, 253-258. https://doi.org/10.3145/epi.2014.may.05

Ure, M. (2016). La comunicación de la administración pública en las redes sociales: los casos de las ciudades de Buenos Aires y Bolonia. Palabra Clave, (19)1.

\section{AUTORES}

\section{Emilia Smolak Lozano}

Licenciada en Sociología por la Universidad Jaguellónica (Polonia) y Estudios Europeos (Universidad de Exeter, Reino Unido). Máster en Gestión Estratégica e Innovación y Doctora, con el premio de doctorado, en ciencias de la comunicación por la Universidad de Málaga donde desarrolla su labor docente e investigador en el campo de las relaciones públicas y publicidad con un interés particular enfocado en la comunicación digital, social media, analítica web y análisis semántico. Autora de los libros sobre la comunicación política y campañas digitales y más de 50 artículos y capítulos de libros sobre la temática de redes sociales. Ponente en más de 50 congresos internacionales. Profesora visitante en la Universidad de Bayreuth e investigadora en estancias en varias universidades europeas.

Orcid ID: https://orcid.org/0000-0001-8193-8786

\section{Ana Almansa-Martínez}

Profesora Titular de Universidad, en el Departamento de Comunicación Audiovisual y Publicidad, Universidad de Málaga (España). Autora de libros como Del gabinete de prensa al gabinete de comunicación, Nuevos medios en Comunicación Política, Assessorías de Comunicaçao y de más de cincuenta artículos en revistas científicas. 
Investigadora principal en el proyecto de investigación Lobby y Comunicación en España (Programa Nacional de I+D), La Comunicación de los bienes patrimoniales para el desarrollo económico y social de Andalucía (Centro de Estudios Andaluces) y del proyecto Observatorio de los Gabinetes de Comunicación en Andalucía (Proyecto de Excelencia). Directora de 25 tesis doctorales ya defendidas. Editora de la Revista Internacional de Relaciones Públicas. Coordinadora del Máster Oficial Dirección Estratégica e Innovación en Comunicación.

Orcid ID: https://orcid.org/0000-0003-0256-6369

Google Scholar: https://scholar.google.es/citations?user=Q8LWr1AAAAAJ\&hl=ca

ResearchID: https://publons.com/researcher/F-8339-2016/ 